\title{
Reduced Modeling Framework of Circulatory System Revisited
}

\author{
Yashar Seyed Vahedein, Alexander S. Liberson \\ Rochester Institute of Technology \\ James E. Gleason Building, 76 Lomb Memorial Drive \\ Rochester, NY 14623-5604, United States \\ yashar.seyed.vahedein@rit.edu; asleme@ rit.edu
}

\begin{abstract}
A modified reduced fluid-structure interaction model is derived based on expanded Hamilton's variational principle governing the coupled incompressible viscous fluid - structure interaction (FSI) in a compliant bifurcated network. To enforce the continuity equation a Lagrange multiplier is utilized which in case of an incompressible fluid coincides with fluid pressure. The first variation of an expanded action functional yields the nonlinear governing Euler-Lagrange equations for the fully coupled nonlinear fluid - structure problem with account for fluid gravity potential. The correct boundary conditions are specified at junctions as natural boundary conditions following from the variational principle. The hyperbolic properties of derived mathematical model are analyzed and used, constructing the monotone finite volume numerical scheme, second-order accuracy in time and space. The accuracy of applied TVD (total variation diminishing) and Lax-Wendroff methods are analyzed by comparison of numerical results to the available analytical smooth and discontinuous solutions.
\end{abstract}

Keywords: Hamilton's variational principle, incompressible viscous flow, reduced fluid-structure interaction (FSI), bifurcated arterial networks, total variation diminishing method (TVD), Lax-Wendroff methodt, break-down solution

\section{Nomenclature}

\begin{tabular}{lllr} 
PWV & Pulse wave velocity $(\mathrm{m} / \mathrm{s})$ & $\mathrm{R}, \mathrm{r}$ & \multicolumn{1}{c}{ Internal wall radii in a zero } \\
FSI & Fluid structure interaction & stress and loaded conditions respectively $(\mathrm{m})$ \\
$\mathrm{A}$ & Cross sectional area $(\mathrm{m} 2)$ & $\eta$ & Ratio of the wall deflection to \\
$\mathrm{V}$ & Velocity vector $(\mathrm{m} / \mathrm{s})$ & $\mathrm{R}$ & \\
$\mathrm{u}$ & Displacement vector $(\mathrm{m})$ & $\mathrm{c}$ & Moens-Korteweg speed of \\
$\mathrm{p}$ & Pressure $(\mathrm{Pa})$ & propagation $(\mathrm{m} / \mathrm{s})$ & \\
$\rho$ & Density of incompressible fluid & $\sigma, \tau$ & Axial normal and shear viscous \\
& $(\mathrm{kg} / \mathrm{m} 3)$ & stress $(\mathrm{Pa})$ & \\
$\mathrm{U}$ & Internal Energy $(\mathrm{J})$ & $v$ & Kinematic viscosity $(\mathrm{m} 2 / \mathrm{s})$
\end{tabular}

\section{Introduction}

Modeling of internal flows through compliant vessels and circulatory networks has been studied intensively for decades with applications to cardiovascular and cerebrospinal circulation. A historical review of arterial fluid mechanics models was presented by Parker - 2009 [1]. Detailed derivation of simplified reduced FSI models for a linear elastic arterial system with account of visco-elasticity and inertia of the wall can be found in Formaggia et al. - 2009 [2]. Physical nonlinearity of thin and thick walls coupled with large deformations have been introduced in FSI dynamics by Liberson et al. - 2016 [3] and Lillie et al. - 2016 [4]. The variety of models and different numerical solvers have been introduced based on different of approaches [1 -5]. We demonstrate the effectiveness of Hamiltonian variational principle in analyzing FSI without any limitations on dissipative fluid dynamics and physical properties of an adjacent flow path wall. We are using the Lagrangian multiplier, which has a connotation of pressure, accounting for the continuity equation of incompressible flow. The Euler-Lagrange equations, being averaged according to the Hamilton's action functional, serve as a generalized quasi-one-dimensional mathematical model for the effective momentum equation

The variational approach, yielding governing equations of physical phenomena, serves as an indispensable tool in case, when the interaction of the system components are non-trivial, containing, as an example, strong nonlinearities, kinematic constraints, high derivatives. The book of Berdichevsky - 2010 [6] presents a variety of variational principles applied separately to fluids and solids. Kock and Olson - 1991 [7] developed a variational approach for FSI system, restricting 
analysis by a linear elastic thin-walled cylinder and an inviscid, irrotational and isentropic fluid flow. Lagrangian multipliers are used to reinforce continuity equation and boundary conditions. Multiple references can be found in this paper relating to applications of the variational approach to the analysis of small vibrations of elastic bodies in a potential fluid.

Numerical effectiveness in a simulation of a pulsating flow is characterized by its ability to track a propagating wave for a few periods without suffering from numerical dissipation (errors in amplitude) and numerical dispersion (artificial oscillations). The most popular numerical methods in this area are the Lax-Wendroff finite volume method, its TaylorGalerkin finite element counterpart, and a discontinuous Galerkin spectral finite element method [2]. We demonstrate superiority in accuracy, for the second order approximation, TVD method [8-10], which could be essential when simulating a model with discontinuity in the load, including its derivatives, or material properties.

As an extension of our previous work [11], here we account for the variational formulation for an incompressible flow subject to the effects of gravity. To enforce the continuity equation a Lagrange multiplier is utilized which in case of an incompressible fluid coincides with fluid pressure. Nonlinear compliant wall model accounts for the transverse shear deformation, which is important for the thick walled or the low shear modulus vessels. Due to this consideration, the derived formulation has applications in both circulatory and cerebrospinal flow simulations.

\section{The Variational Principle for Fluid-Structure Interaction Problems}

Hamilton's variational principle is enunciated as a universal principle of nature unifying mechanical, thermodynamic, electromagnetic and other fields in a single least action functional, subject to extremization for a true process. According to the mentioned principle, the action functional $I$ being applied to FSI problem

$$
I=I_{\text {fluid }}+I_{\text {solid }}=\int_{t 1}^{t 2}\left[\oint_{\forall \text { fluid }(t)} \rho_{f} L_{f} d \forall+\oint_{\forall_{\text {solid }(t)}} L d \forall\right] d t=0
$$

possesses a stationary value under the additional constraint of a continuity. Here $\delta I_{\text {fluid }}, \delta I_{\text {solid }}$ are variations of action components across fluid and solid volumes $\forall_{\text {fluid }(t)}, \forall_{\text {solid }(t)} ; \mathrm{t}$ - time, $\rho_{f}$-density of the fluid, $L_{f}, L$ - the Lagrangian density functions for fluid and solids respectfully.

\subsection{Fluid Domain}

As it is mentioned by Berdichevsky - 2010 [6], variation of the Lagrange function density in Eulerian coordinates can be written as follows:

$$
\delta L_{f}=\delta\left(\frac{\boldsymbol{V}^{2}}{2}-U(S, \nabla \mathbf{u})-\Phi(\mathbf{u})\right)+T \delta S
$$

where $\boldsymbol{V}$ - is a velocity vector, $U$ - is an internal energy as a function of entropy $S$, and a distortion tensor $\boldsymbol{\nabla} \mathbf{u}$ (gradient of a displacement vector $\mathbf{u}$ ), T - temperature, $\Phi=\rho_{f} g \boldsymbol{u} \cdot \boldsymbol{l}_{g}$-gravity potential, $\boldsymbol{l}_{g}$ - unit vector of gravity. Velocity and the displacement vector are not the subjects to independent variations.

According to the variational principle, the functional (1) possesses a stationary value under the additional constraint

$$
\boldsymbol{\nabla} \cdot \boldsymbol{u}=\mathbf{0}
$$

Presenting variation of a velocity as a substantial derivative of a variation of a displacement vector, arrive at:

$$
\delta \boldsymbol{V}=\frac{D \delta \boldsymbol{u}}{D t}=\frac{\partial \delta \boldsymbol{u}}{\partial t}+\boldsymbol{V} \cdot \boldsymbol{\nabla} \delta \boldsymbol{u}
$$


Now we have reduced the minimization problem to the only two independent variables - displacement and entropy. Removing constraint (3) by using Lagrangian multiplier P, with account for identity for the deviatoric stress tensor $\boldsymbol{\sigma}=$ $\rho_{f} \frac{\partial U}{\partial \nabla \boldsymbol{u}}$, obtain from (2)-(4)

$$
\delta I_{\text {fluid }}=\int_{t 1}^{t 2}\left[\oint_{\forall \text { fluid }(t)}\left(\rho_{f} \boldsymbol{V} .\left(\frac{\partial \delta \boldsymbol{u}}{\partial t}+\boldsymbol{V} \cdot \boldsymbol{\nabla} \delta \boldsymbol{u}\right)+(P \boldsymbol{\nabla}-\boldsymbol{\nabla} \Phi) \cdot \delta \boldsymbol{u}-\rho_{f}\left(\frac{\partial U}{\partial s}-T\right) \delta s-\boldsymbol{\sigma}: \delta \boldsymbol{\nabla u}\right) d \forall\right] d t
$$

Considering 2D axisymmetric flow in a long compliant tube, according to the long wave approximation we neglect variability of a radial velocity component and a pressure in a radial direction. The equation (5) in this case is transformed to the following form:

$$
\delta I_{\text {fluid }}=\int_{t 1}^{t 2} \int_{x}^{R(x, t)} \int_{0}^{R}\left[\rho_{f} V\left(\frac{\partial \delta u}{\partial t}+V \frac{\partial \delta u}{\partial x}\right)+P \frac{\partial \delta u}{\partial x}-\sigma \frac{\partial \delta u}{\partial x}-\tau \frac{\partial \delta u}{\partial r}-\rho_{f} g l_{g x}\right] r d r d x d t
$$

Here $V$-is an axial velocity, $u$-is an axial component of displacement, $\sigma, \tau$ - axial normal and shear viscous stress components, $R(x, t)$ - internal radius of a tube as a function of axial coordinate and time, $l_{g x}$. - cosine of the angle between $\mathrm{g}$ and $\mathrm{x}$ directions. The reduced models are based on assumptions regarding radial profiles, i.e.

$$
V(x, r, t)=\varphi(r) \bar{V}(x, t) ; \quad u(x, r, t)=f(r) \bar{u}(x, t)
$$

With the aim of application to the incompressible flow, density is assumed constant. Integrating the functional (7) over the cross section with the following integration by parts, arrive at the reduced momentum equation

$$
\frac{\partial \bar{V}}{\partial t}+\frac{\partial}{\partial x}\left(a_{1} \frac{p}{\rho}+a_{2} \bar{V}^{2}\right)=\frac{1}{a_{0} \rho}\left[\int r f(r) \sigma(x, r, t) d r-R \tau(x, R, t)\right]
$$

Where the coefficients are:

$$
a_{0}=\int r f(r) d r ; \quad a_{1}=\int r \varphi(r) f(r) d r ; \quad a_{2}=\frac{1}{a_{0}} \int r \varphi(r)^{2} f(r) d r
$$

In case of Newtonian fluid ( $\sigma=2 \rho v \frac{\partial V}{\partial x}, \tau=\rho v \frac{\partial V}{\partial r}$ ), generalized Hagen-Poiseuille profile $\varphi(r)=\frac{\gamma+2}{\gamma}\left[1-\left(\frac{r}{R}\right)^{\gamma}\right]$ and a constant profile for the function distribution in radial direction, $f(r)=1$, equation (8) takes the form presented by San and Staples - 2012 [13].

$$
\frac{\partial \bar{V}}{\partial t}+\frac{\partial}{\partial x}\left(\alpha \frac{\bar{V}^{2}}{2}+\frac{P}{\rho}\right)-g l_{g x}=v\left(\frac{\partial^{2} \bar{V}}{\partial x^{2}}-2(\gamma+2) \frac{\bar{V}}{R^{2}}\right)
$$

Besides equation (8), Hamilton's equation in a form of $\delta I_{\text {fluid }}=0$ yields natural boundary conditions. In case of a multiscale model, matching section of a coupled 3D and 1D require continuity following from natural boundary conditions

$$
a_{1} \frac{p}{\rho}+a_{2} \bar{V}^{2}=\int r f(r)\left(\frac{p}{\rho}+V^{2}\right) d r
$$


It should be noted that we neglect the effect of dissipation on boundary conditions.

\subsection{Solid Domain}

Consider a circular thin-wall cylinder relating to the polar system of coordinates. Let $R$ be the radius of the wall under the load, $R_{0}$ - radius in a load free state, $h$ - the wall thickness, $\lambda_{\theta}=R / R_{0}$ - circumferential stretch ratio, $\eta=\left(\lambda_{\theta}-1\right)-$ nondimensionalized wall normal displacement. Introducing wall kinetic energy $K$, elastic energy $U_{e l}$ and a dissipative energy $U_{d}$ and work of external load $W_{p}$ the Hamiltonian functional relating to the solid domain can be presented as

$$
\delta I_{\text {solid }}=\iint\left(\delta K-\left(\delta U_{e l}+\delta U_{d}-\delta W_{p}\right)\right) d x d t
$$

Kinetic energy per unit length is defined by the normal velocity of the moving wall $R_{0} \frac{d \eta}{d t}$

$$
K=\frac{1}{2} \rho h R_{0}^{2}(1+\eta)\left(\frac{\partial \eta}{\partial t}\right)^{2}
$$

Internal elastic energy is composed of hyperelastic exponential membrane strain energy (Fung, 1990) [12], transverse shear strain energy due to the finite transverse shear strain modulus $\mathrm{G}$, and an energy, accumulated by a longitudinal prestress force $\mathrm{N}$ per unit area

$$
U_{e l}=\frac{c}{2}\left(e^{Q}-1\right)+\frac{1}{2} G R_{0}^{2}\left(\frac{\partial \eta}{\partial x}\right)^{2}+N\left(\sqrt{1+R_{0}^{2}\left(\frac{\partial \eta}{\partial x}\right)^{2}}-1\right)
$$

Where $Q=a_{11} E_{\theta}^{2}+2 a_{12} E_{\theta} E_{z}+a_{22} E_{z}^{2}$, and $c, a_{11}, a_{12}, a_{22}$ are material constants from Fung et al. anisotropic model [12]. Assuming the wall model is a system of independent nonlinearly elastic rings, and simplifying the equation (14) by leaving the principle quadratic terms only (the forth power for $\eta$ and quadratic terms for the slope), arrive at

$$
U_{e l}=\frac{c a_{11}}{8}\left(\eta^{4}+4 \eta^{3}+4 \eta^{2}\right)+\frac{(N+G)}{2} R_{0}^{2}\left(\frac{\partial \eta}{\partial x}\right)^{2}
$$

Elementary work produced by the viscous component of circumferential stress relating to the Voight type of material and external pressure load are presented as

$$
\delta U_{d}-\delta W_{p}=\left(\frac{\mu h}{R_{0}} \frac{\partial \eta}{\partial t}-p\right) R_{0} \delta \eta
$$

Substituting (13)-(16) into equation (12), and equating to zero, obtain the equation of motion of an axisymmetric cylinder in the following explicit form with respect to pressure

$$
p=\rho h R_{0} \frac{\partial^{2} \eta}{\partial t^{2}}+\frac{\mu h}{R_{0}} \frac{\partial \eta}{\partial t}+c a_{11}\left(\frac{3}{2} \eta^{2}+\eta\right)-(N+G) R_{0}{ }^{2} \frac{\partial^{2} \eta}{\partial x^{2}}
$$

Momentum equation (8), equation of a boundary wall motion (17) and an averaged over the cross-section continuity equation (3)

$$
\frac{\partial A}{\partial t}+\frac{\partial}{\partial x}(\bar{V} A)=0 ; \quad \mathrm{A}=(\eta+1)^{2}
$$


create a closed-form reduced mathematical model for fluid-structure interaction in a compliant channel.

\section{Numerical Simulation}

Numerical solvers utilized in a computational hemodynamics are typically based on the Lax-Wendroff scheme, its Taylor-Galerkin finite element counterpart, or a discontinuous Galerkin spectral finite element method [13]. In this paper the second-order accuracy monotonicity preserving TVD scheme is presented, which demonstrates its superiority for the problems dealing with a discontinuity of a load function, including discontinuity of its derivative, or material properties. With application to the following basic equation

$$
\frac{\partial Q}{\partial t}+\frac{\partial F(Q)}{\partial x}=0
$$

a conservative monotonicity preserved approximation of unknown vector $Q$ reads [ 8 ]

$$
\begin{aligned}
& Q_{j}^{\mathrm{n}+1}=Q_{\mathrm{j}}^{n}-\frac{\Delta x}{\Delta t}\left(F_{i+\frac{1}{2}}^{n+\frac{1}{2}}-F_{i-\frac{1}{2}}^{n+\frac{1}{2}}\right) \\
& F_{i+\frac{1}{2}}^{n+\frac{1}{2}}=A^{+} Q_{j+1}^{n}+A^{-} Q_{j}^{n}+\widetilde{F}_{i+\frac{1}{2}}^{n+\frac{1}{2}} \\
& \tilde{F}_{i+\frac{1}{2}}^{n+\frac{1}{2}}=\frac{1}{2}|A|\left(I-\frac{\Delta x}{\Delta t}|A|\right) R \Delta \widetilde{W}_{i-1 / 2}, \quad \Delta \widetilde{W}_{i-1 / 2}=\operatorname{diag}(\Psi) \Delta W_{i-1 / 2}
\end{aligned}
$$

Here fractional index corresponds to the numerical cell edge, $R$ - is the modal matrix, i.e. the matrix, composed of eigenvectors of the Jacobian matrix $A=\partial F / \partial Q, \Delta W_{i-1 / 2}=W_{i}-W_{i-1}$, in which $W_{i}$ is the vector of characteristic

variables , $\Psi=\left(\psi_{i}^{p}\right)$ - vector of "limiters", preserving monotonicity of a numerical scheme, p - index of the current eigenvalue

$$
\left.\psi^{p}=\max ([0, \min (2 r, 1)), \min (2, r)]\right), \quad r=\frac{W_{i+1}^{p}-W_{i}^{p}}{W_{i}^{p}-W_{i-1}^{p}}
$$

The details of TVD methods applied to the system of hyperbolic equations can be found in [8]. Following are two examples, showing the superior behavior of the TVD scheme compared to the common schemes such as Lax-Wendroff and Beam-Warming.

\subsection{Introducing Sine Shape Pulse with Undershoot in the Parent Vessel}

Schematic of a symmetric bifurcated structure with a single parent vessel and two daughter vessels are presented in Figure 1. A single pulse with an undershoot is introduced at $X=0.21 \mathrm{~m}$ along the axis of the parent vessel (vessel 1). Using the TVD scheme, applied to the linearized model [11], the propagation of the forward moving wave subject to the noreflected boundary conditions, is simulated throughout vessels 1,2 and 3 . The complete physical time for this process was $t=0.0312 \mathrm{~s}$. Results presented in Fig. 1-3 correspond to the following input data: density $\rho=0.25 \frac{\mathrm{kg}}{\mathrm{m}^{3}}$, Moens-Korteweg speed of propagation $c=2 \frac{\mathrm{m}}{\mathrm{s}}$, initial velocity profile $(\mathrm{m} / \mathrm{sec})$

$$
V=30 \times\left(\sin \left(\frac{\pi}{0.01} \times(X-0.21)\right)+\cos \left(0.25 \times \frac{\pi}{0.01} \times(X-0.21)\right)\right)
$$



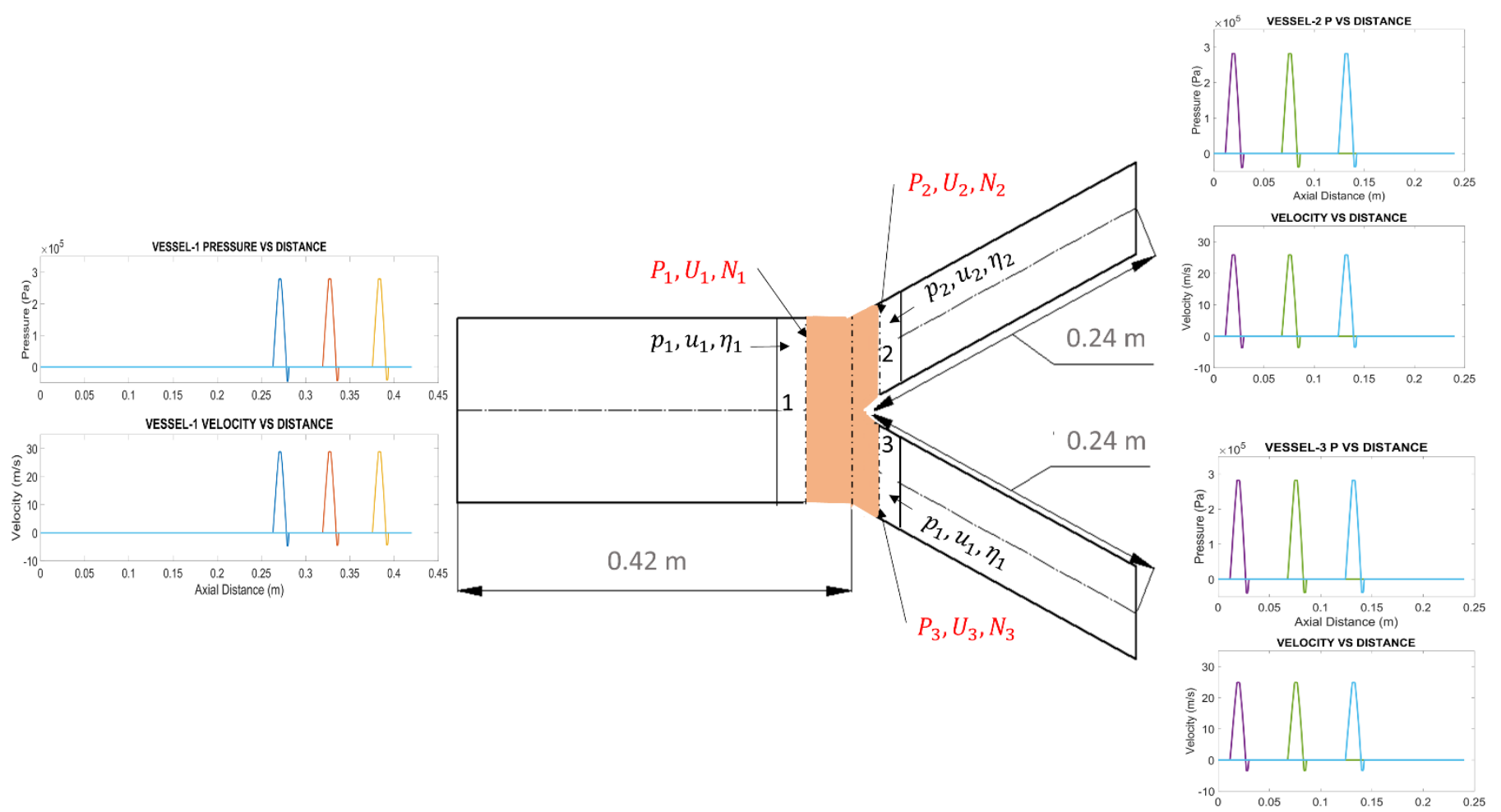

Fig. 1: Schematics of the bifurcated vessels and the pulse wave propagation in each vessel captured by TVD scheme.

As shown in this figure, the pressure and velocity waveform keep their shape and magnitude propagating down from vessel 1 and passing junctions with vessels 2 and 3. Figure 2 illustrates comparison of results obtained by TVD scheme with ones predicted by the Lax-Wendroff (L-W) and Beam-Warming (B-W) methods. Both L-W and B-W schemes are the finite volume methods second order of accuracy in time and space. As shown in Fig.2 efficiently approximate the wave in the continuous regions, but show spurious numerical oscillations at the onset and after the wave undershoot, respectively. The latter indicates that L-W and B-W schemes are essentially dispersive schemes. In contrast to L-W and B-W, the TVD method preserves the shape of the wave in both continuous and discontinuous regions of the pulse wave. 

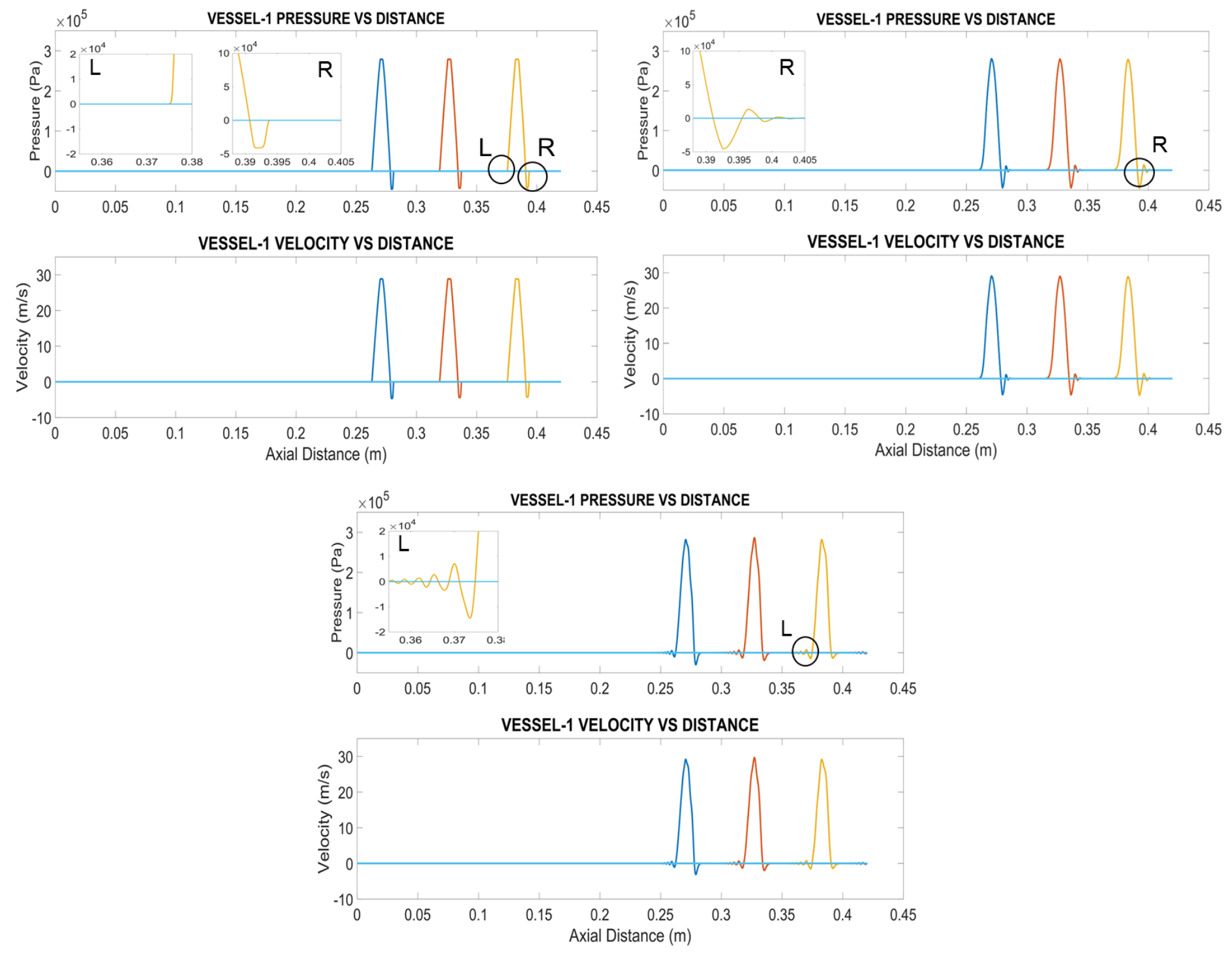

Fig. 2: From left to right and top to bottom. Comparison of TVD; Beam-Warming; and Lax-Wendroff numerical methods in capturing the pressure and velocity sin wave (with undershoot) propagation in Vessel $1(t=0.0312 \mathrm{~s})$. 


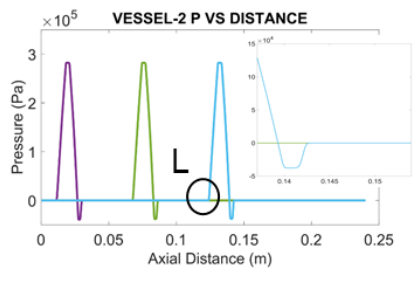

VELOCITY VS DISTANCE

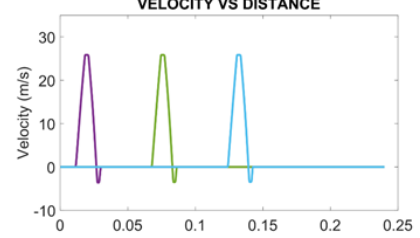

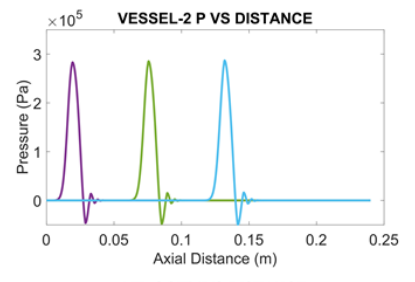

VELOCITY VS DISTANCE
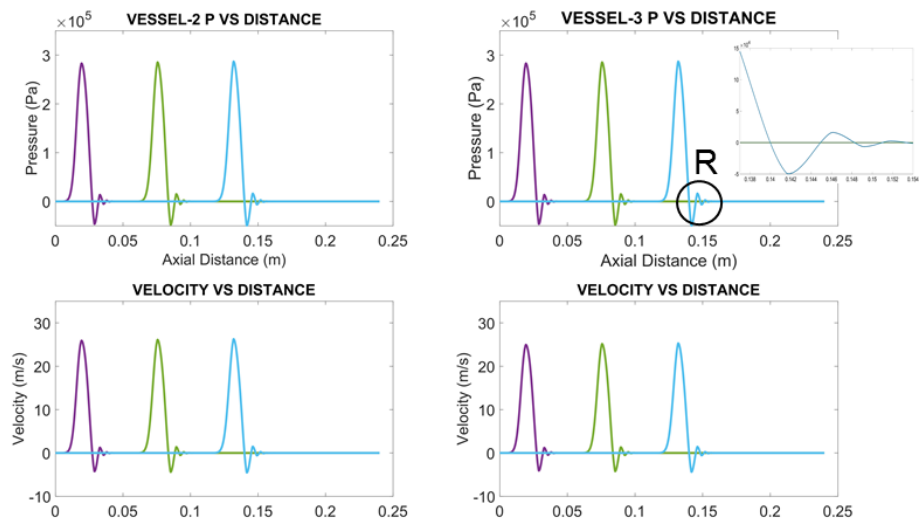
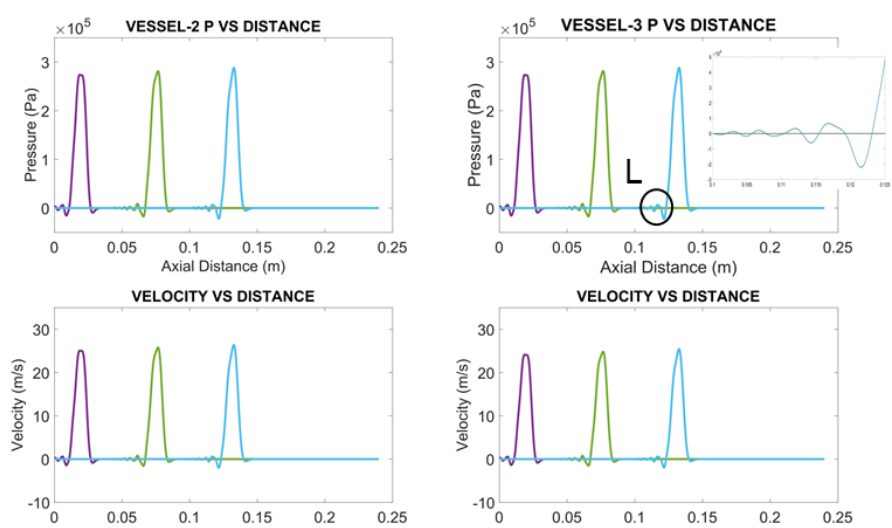

Fig. 3: From left to right and top to bottom: Comparison of TVD; Beam-Warming; and Lax-Wendroff numerical methods in capturing the pressure and velocity sin wave (with undershoot) propagation in Vessels 2 and $3(t=0.0312 \mathrm{~s})$.

In the zoomed in pictures from each figure, the oscillations (dispersion) in B-W and L-W compared to TVD method can be seen more clearly.

\subsection{Introducing Rectangular Pulse in Parent Vessel}

A rectangular pulse in the middle of the parent vessel is introduced. The following properties are held the same as in the section 3.1: $\rho=0.25 \frac{\mathrm{kg}}{\mathrm{m}^{3}}$ and $c=2 \frac{\mathrm{m}}{\mathrm{s}}$. The step size is $0.02 \mathrm{~m}$ and it starts at $X=0.21 \mathrm{~m}$. We compare the results of TVD scheme with Lax-Wendroff (L-W) and Beam-Warming (B-W) methods. Since the difference between the right and left side of the discontinuity is more severe, the dispersion effect manifets itself more clearly for B-W and L-W methods. As expected TVD scheme does not show any sign of dispersive effect and remains monotone. Any topological and material related alterations can cause dispersion in the circulatory system. Therefore, it is important to capture the shape of a moving pressure/velocity waveform without the loss in magnitude or monotonicity. 

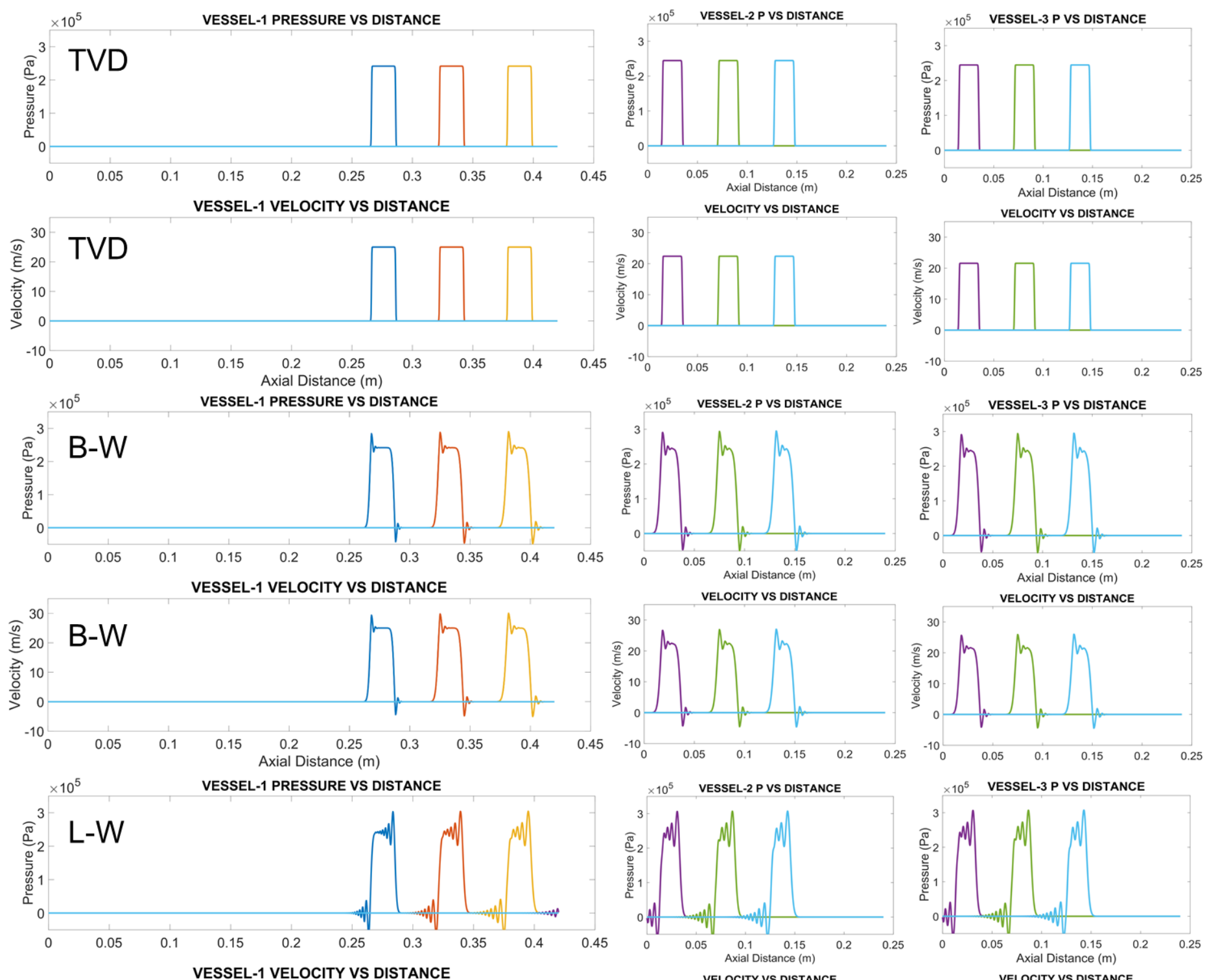

$\times 10^{5}$ VESSEL-3 P VS DISTANCE
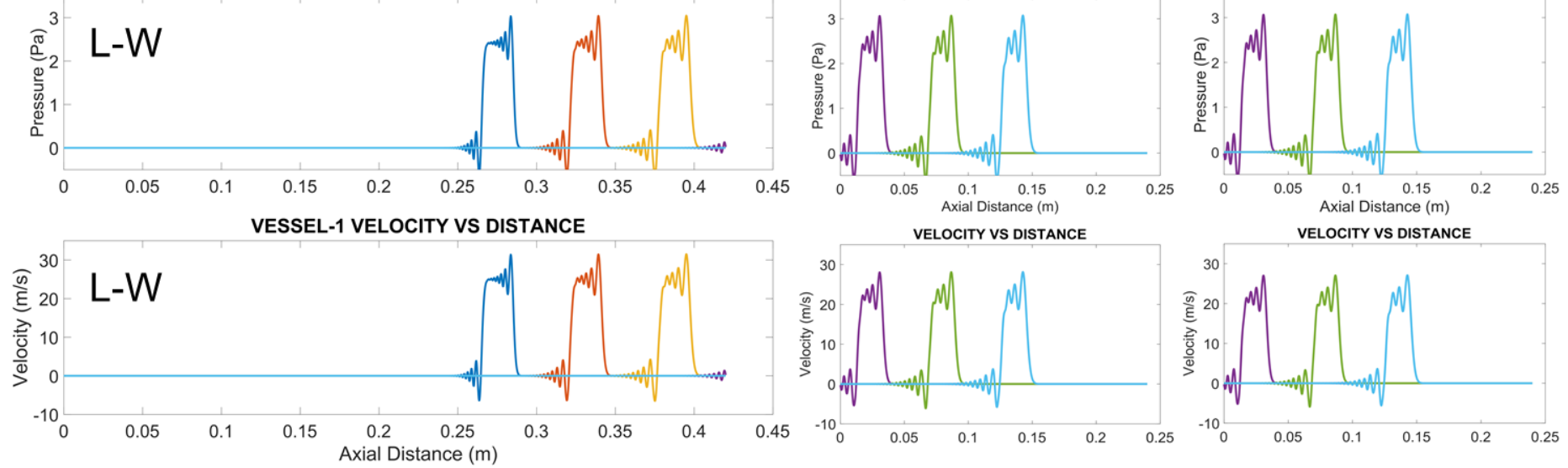

Fig. 4: From left to right and top to bottom: Comparison of TVD; Beam-Warming; and Lax-Wendroff numerical methods in capturing the pressure and velocity rectangular wave propagation in Vessels 1,2 and $3(t=0.0312 s)$.

The oscillatory behavior (in L-W and B-W) is amplifying as the pulse moves forward. In a circulatory system with variable vessel wall and compliance, these oscillations can lead to major errors in local predictions of pressure and velocity of the blood.

\section{Conclusions}

A general approach to derive the fluid-structure interaction problem have been applied based on Hamilton's variational principle. Fluid is assumed incompressible and viscous, with a boundary wall - nonlinear viscoelastic. Continuity is imposed as a constraint using the Lagrange multipliers. Numerical results based on a TVD approach are compared to the 
solutions provided by the Lax-Wendroff and Beam-Warming numerical techniques. It is proved that the Lax-Wendroff and Beam-Warming methods are clearly dispersive, providing artificial oscillations when simulating physical problems with discontinuity. These oscillations are not presented when using the TVD method, making it the optimum choice in solving 1D FSI problem.

\section{References}

[1] K. H. Parker, "A brief history of arterial wave mechanics. Medical and Biological Engineering and Computing," vol. 47, no. 2, pp. 111-118, 2009.

[2] L. Formaggia, A. Quarteroni, A. Veneziani, "Cardiovascular Mathematics, of MS\&A, — Modeling, Simulation and Applications," vol. 1, Springer Milan, 2009.

[3] A. S. Liberson, J. S. Lillie, D. A. Borkholder, "A physics based approach to the pulse wave velocity prediction in compliant arterial segments," Journal of Biomechanics, 2016.

[4] J. S. Lillie, A. S. Liberson, D. A. Borkholder, "Quantification of Hemodynamic Pulse Wave Velocity Based on a Thick Wall Multi-Layer Model for Blood Vessels," Journal of Fluid Flow Heat and Mass Transfer, vol. 3, pp. 5461, 2016.

[5] A. S. Liberson, J. S. Lillie, D. A. Borkholder, "Shock Capturing Numerical Solution for the Boussinesq Type Models with Application to Arterial Bifurcated Flow," in Procedings of the International conference on New Trends in Transport Phenomena, Ottawa, Canada, 2014.

[6] V. Berdichevsky, Variational Principles of Continuum Mechnaics, 2010.

[7] E. Kock, L. Olson, "Fluid-structure interaction analysis by the finite element method - a variational approach," International Journal for Numerical Methods in Engineering, vol. 31, 463-491, 1991.

[8] R. Leveque, Finite Volume Methods for Hyperbolic Problems. Cambridge, University press, 2002.

[9] Y. Kosolapov, A. Liberson, "An implicit relaxation method for computation of 3D flows of spontaneously condensing steam," Computational mathematics and mathematical physics, vol. 37, no. 6, pp. 759-768, 1997.

[10] A. Liberson, S. Hesler, T. Mc.Closkey, "Inviscid and viscous numerical simulation for non-equilibrium spontaneously condensing flows in steam turbine blade passages. PWR-Vol.33," International Joint Power Generation Conference, vol. 2, ASME, pp. 97-105, 1998.

[11] A. S. Liberson, Y. Seyed Vahedein D. A. Borkholder, "Variational Approach of Constructing Reduced FluidStructure Interaction Models in Bifurcated Networks," in Proceedings of the 2nd World Congress on Momentum, Heat and Mass Transfer (MHMT'17), Spain, 2017. DOI: 10.11159/enfht17.103

[12] Y. C. Fung, K. Fronek, P. Patitucci, "Pseudoelasticity of arteries and the choice of its mathematical expression," Am J Physiol., vol. 237, no. 5, pp. H620-31, 1979.

[13] O. San, A. Staples, "An improved model for reduced order physiological fluid flows," Journal of Mechanics in Medicine and Biology, pp. 1-37, 2012. 